\title{
Transmitter Preprocessing Aided Spatial Modulation for Multiple-Input Multiple-Output Systems
}

\author{
Lie-Liang Yang \\ School of ECS, University of Southampton, SO17 1BJ, United Kingdom \\ Tel: 0044-(0)23-8059 3364, Email:1ly@ecs.soton.ac.uk, http://www-mobile.ecs.soton.ac.uk
}

\begin{abstract}
This paper proposes and studies a transmitter preprocessing aided spatial modulation (PSM) scheme, which conveys information jointly by a conventional amplitude-phase modulation (APM) and a preprocessing aided space shift keying (pre-SSK) modulation. In contrast to the existing SSK modulation, which carries information using the indexes of transmitter antennas and assumes channel state information at receiver (CSIR), the preSSK modulation extracts the transmitted information using the indexes of receive antennas and assumes channel state information at transmitter (CSIT). This paper addresses the issues of preprocessing optimization and detection of PSM signals. Furthermore, the bit-error-rate (BER) performance of the PSM is investigated, when assuming that the channel from any transmit antenna to any receive antenna experiences independent Rayleigh fading. Our studies show that, when appropriately designed, the pre-SSK and APM invoked can enhance each other, resulting in that the PSM is capable of attaining a better BER performance than the corresponding pure pre-SSK and pure APM.
\end{abstract}

Index Terms-Multiple-input multiple-output, spatial modulation, space shift keying, preprocessing, detection.

\section{INTRODUCTION}

Recently, space-based modulation has been proposed for alternative signal transmission in multiantenna multiple-input multiple-output (MIMO) wireless systems [1-8]. With the space-based modulation, information is conveyed either jointly by a conventional amplitude-phase modulation (APM), such as phase-shift keying (PSK), quadrature amplitude modulation (QAM), etc., and the indexes of transmit antennas, forming the so-called spatial modulation (SM) [2-4, 8], or solely by the indexes of transmit antennas, referred to as the space shift keying (SSK) modulation [5,6]. Explicitly, the SSK modulation is a special case of the more general SM scheme. The studies show that the SM and SSK modulation schemes employ some advantages over the conventional MIMO schemes, such as BLAST and space-time block coding (STBC) [9-11]. As argued in $[2-6,8]$, due to activating only one antenna for transmission, the SM and SSK modulation schemes are capable of avoiding the inter-channel interference and reducing the complexity of detection. Furthermore, due to using single transmit antenna, the SM and SSK modulation schemes do not require interantenna synchronization as in the BLAST or STBC systems.

The SM and SSK modulation have been investigated from different perspectives, as illustrated in [2-8]. An excellent review in terms of the history, studies and advancement of spacebased modulation can be found in [7], which are therefore not repeated here. The reader who is interested in the details is referred to [7] and the references there in, as well as the other references mentioned above.
So far, the space-based modulation schemes have been studied, when assuming that information is carried by the indexes of transmit antennas under the assumption that channel state information (CSI) is employed at receiver, i.e., under the CSIR mode. In MIMO communications, there are some cases where CSI is preferably to be exploited at transmitter side, which is often referred to as the CSIT mode, in order to reduce the complexity of receiver [12]. Furthermore, it is well recognized that the MIMO systems operated under CSIT mode have a range of advantages over that operated under CSIR mode [13]. First, when a MIMO system has more transmit antennas than receive antennas, then, its capacity when operated under the CSIT mode may be much higher than that when operated under the CSIR mode $[12,13]$. Second, in contrast to the openloop space-time diversity (STD) schemes operated under CSIR mode, which can only attain the transmit diversity gain, the closed-loop STD schemes under CSIT mode are capable of providing the receiver with the signal-to-noise ratio (SNR) gain, in addition to the promised transmit diversity gain [12].

In this contribution, we propose and investigate a spacebased modulation scheme, namely the preprocessing aided spatial modulation (PSM) scheme. The proposed PSM uses the indexes of receive antennas as a constellation to carry information, in additional to a conventional APM scheme. To be more specific, a PSM symbol consists of two components of information, one component is conveyed by the indexes of receive antennas, referred to as the pre-SSK, and the other by the conventional APM. The PSM uses certain transmitter preprocessing scheme to identify a desired receive antenna, based on which the detector can extract the first component of information carried in the spatial domain. The second component of information is recovered from the conventional APM invoked. Explicitly, the proposed PSM can be viewed as a dual modulation scheme of the SM for MIMO communications, as investigated in [1-8]. Therefore, it is highly important to study the theory, implementation, achievable performance, etc., of the PSM. Specifically, in this contribution, we illustrate the principles, consider the preprocessing optimization and detection, as well as study the achievable bit-error-rate (BER) performance of the PSM. From our studies and performance results, we find that, when properly designed, the pre-SSK modulation and conventional APM invoked can enhance each other's BER performance, making the PSM achieve a better overall BER performance than the corresponding pure pre-SSK modulation and pure APM.

The rest of the paper is organized as follows. In Section II, the principles of PSM is described. Section III considers the 
transmitter preprocessing, while Section IV addresses the detection of PSM signals. Performance results are illustrated and discussed in Section V. Finally, conclusions are stated in Section VI.

\section{System Model}

The MIMO system addressed employs $N$ transmit antennas and $M_{1}=2^{k_{1}}$ receive antennas, where $N \geq M_{1}$ is assumed. The $\left(N \times M_{1}\right)$ channel matrix is expressed as $\boldsymbol{H}=\left[\boldsymbol{h}_{0}, \boldsymbol{h}_{1}, \cdots, \boldsymbol{h}_{M_{1}-1}\right]$, where $\boldsymbol{h}_{m_{1}}, m_{1}=0, \ldots, M_{1}-1$, is an $N$-length column vector containing the channel gains from the $N$ transmit antennas to the $m_{1}$ th receive antenna. We assume that the channel knowledge is only known to the transmitter, not known to the receiver. Let the transmitter preprocessing matrix be expressed by $\boldsymbol{P}=\left[\boldsymbol{p}_{0}, \boldsymbol{p}_{1}, \cdots, \boldsymbol{p}_{M_{1}-1}\right]$, which is an $\left(N \times M_{1}\right)$ matrix that is designed according to certain optimization criterion, as will be considered later in Section III. The preprocessing matrix $\boldsymbol{P}$ is normalized to satisfy $\operatorname{Tr}\left(\boldsymbol{P} \boldsymbol{P}^{H}\right)=M_{1}$, where $\operatorname{Tr}(\cdot)$ denotes the trace of a concerned square matrix. Depended on the $k_{1}$ bits of information transmitted, one of the $M_{1}$ columns of the preprocessing matrix $\boldsymbol{P}$ is activated for transmission, forming the pre- $M_{1} \mathrm{SSK}\left(M_{1}-\right.$ ary SSK) modulation. Let $\mathcal{X}=\left\{X_{0}, X_{1}, \cdots, X_{M_{2}-1}\right\}$, where $M_{2}=2^{k_{2}}$ and $E\left[\left|X_{i}\right|^{2}\right]=1$, be a set of signals belonging to an $M_{2}$-ary constellation of APM, such as $M_{2}$-ary phase-shift keying $\left(M_{2} \mathrm{PSK}\right), M_{2}$-ary quadrature amplitude modulation $\left(M_{2} \mathrm{QAM}\right)$, etc. Then, with our proposed PSM, let us assume that $m_{1} \in \mathcal{M}_{1}=\left\{0,1, \cdots, M_{1}-1\right\}$ is an integer determined by $k_{1}$ binary bits and $x \in \mathcal{X}$ is a baseband signal determined by another $k_{2}$ binary bits. Then, the discrete signal transmitted from the $N$ transmit antennas is given by

$$
\boldsymbol{s}=\boldsymbol{p}_{m_{1}} x
$$

where $\boldsymbol{p}_{m_{1}}$ is the preprocessing vector determined by $m_{1}$. Explicitly, we have $E\left[\|s\|^{2}\right]=1$, i.e., the transmitted signal has unity power, due to the constraints of $\operatorname{Tr}\left(\boldsymbol{P} \boldsymbol{P}^{H}\right)=M_{1}$ and $E\left[\left|X_{i}\right|^{2}\right]=1$. The signaling of (1) conveys in total $\left(k_{1}+k_{2}\right)$ bits of information per symbol.

When $s$ is transmitted over the MIMO channels defined by $\boldsymbol{H}$, the received observation at the $m$ th receive antenna is given by

$$
y_{m}=\boldsymbol{h}_{m}^{T} \boldsymbol{p}_{m_{1}} x+n_{m}, m=0,1, \ldots, M_{1}-1
$$

Let us define

$$
\begin{aligned}
& \boldsymbol{y}=\left[y_{0}, y_{1}, \cdots, y_{M_{1}-1}\right]^{T} \\
& \boldsymbol{n}=\left[n_{0}, n_{1}, \cdots, n_{M_{1}-1}\right]^{T}
\end{aligned}
$$

Furthermore, we define the overall signal set as

$$
\begin{aligned}
\mathcal{S} & =\left\{\mathcal{S}^{(0)}, \mathcal{S}^{(1)}, \cdots, \mathcal{S}^{\left(M_{1}-1\right)}\right\} \\
\mathcal{S}^{\left(m_{1}\right)} & =\left\{\boldsymbol{x}_{0}^{\left(m_{1}\right)}, \boldsymbol{x}_{1}^{\left(m_{1}\right)}, \cdots, \boldsymbol{x}_{M_{2}-1}^{\left(m_{1}\right)}\right\}, m_{1}=0, \ldots, M_{1}-1 \\
\boldsymbol{x}_{m_{2}}^{\left(m_{1}\right)} & =\left[0, \cdots, 0, X_{m_{2}}, 0, \cdots, 0\right]^{T}, m_{2}=0,1, \ldots, M_{2}-1
\end{aligned}
$$

where $\boldsymbol{x}_{m_{2}}^{\left(m_{1}\right)}$ is an $M_{1}$-length vector with $X_{m_{2}}$ being its $m_{2}$ th element and the other elements being zero elements. With the aid of the above definitions, it can be shown that we have

$$
\boldsymbol{y}=\boldsymbol{H}^{T} \boldsymbol{P} \boldsymbol{x}+\boldsymbol{n}
$$

where $x \in \mathcal{S}$. For example, when the transmitted $\left(k_{1}+k_{2}\right)$ bits are mapped to the $M_{1}$ SSK symbol $m_{1}$ and $M_{2}$ PSK or $M_{2}$ QAM symbol $X_{2}$, then we have $\boldsymbol{x}=\boldsymbol{x}_{2}^{\left(m_{1}\right)}$ in (5). Note that, in (5), $n$ obeys the Gaussian distribution with zero mean and an $\left(M_{1} \times M_{1}\right)$ covariance matrix $\sigma^{2} \boldsymbol{I}_{M_{1}}$, where $\sigma^{2}=1 / \gamma_{s}=$ $\left[\left(k_{1}+k_{2}\right) \gamma_{b}\right]^{-1}$ with $\gamma_{s}$ and $\gamma_{b}$ denoting the average signalto-noise ratio (SNR) per symbol and the average SNR per bit, respectively.

\section{Optimization of Transmitter Preprocessing}

Since the receiver has no channel knowledge, the receiver can detect the information transmitted in maximum-likelihood (ML) principles according to the optimization problem

$$
\begin{aligned}
\hat{m}, \hat{x} & =\arg \min _{\boldsymbol{x}_{m_{2}}^{\left(m_{1}\right)} \in \mathcal{S}}\left\{\left\|\boldsymbol{y}-\boldsymbol{x}_{m_{2}}^{\left(m_{1}\right)}\right\|^{2}\right\} \\
& =\arg \max _{\boldsymbol{x}_{m_{2}}^{\left(m_{1}\right)} \in \mathcal{S}}\left\{2 \Re\left\{\boldsymbol{y}^{H} \boldsymbol{x}_{m_{2}}^{\left(m_{1}\right)}\right\}-\left\|\boldsymbol{x}_{m_{2}}^{\left(m_{1}\right)}\right\|^{2}\right\}
\end{aligned}
$$

In order to assist the receiver's detection based on (6) and achieve the best possible error performance, the preprocessing should be designed to maximize the desired term matching to the transmitted signal and minimize the other $\left(M_{1} M_{2}-1\right)$ terms. Therefore, for any given transmitted symbol $\boldsymbol{x}_{j}^{(i)}$, which is formed by the $M_{1}$ SSK symbol $i \in \mathcal{M}_{1}$ and $M_{2}$-ary symbol $X_{j} \in \mathcal{X}$, the optimization of transmitter preprocessing can be described as

$$
\boldsymbol{P}_{o}=\arg \left\{\begin{array}{c}
\max _{\boldsymbol{P}}\left\{2 \Re\left\{\boldsymbol{y}^{H} \boldsymbol{x}_{j}^{(i)}\right\}-\left\|\boldsymbol{x}_{j}^{(i)}\right\|^{2}\right\}, \\
\min _{\boldsymbol{P}}\left\{2 \Re\left\{\boldsymbol{y}^{H} \boldsymbol{x}_{m_{2}}^{\left(m_{1}\right)}\right\}-\left\|\boldsymbol{x}_{m_{2}}^{\left(m_{1}\right)}\right\|^{2}\right\} \\
\quad \text { for all } m_{1} \neq i \text { and } m_{2} \neq j
\end{array}\right.
$$

We assume that the Gaussian noise is circular symmetric. In this case, after applying (5) into (7), the optimization problem can alternatively be expressed as

$\boldsymbol{P}_{o}=\arg \left\{\begin{array}{c}\max _{\boldsymbol{P}}\left\{2 \Re\left\{\left(\boldsymbol{x}_{j}^{(i)}\right)^{H} \boldsymbol{P}^{H} \boldsymbol{H}^{*} \boldsymbol{x}_{j}^{(i)}\right\}-\left\|\boldsymbol{x}_{j}^{(i)}\right\|^{2}\right\}, \\ \min _{\boldsymbol{P}}\left\{2 \Re\left\{\left(\boldsymbol{x}_{j}^{(i)}\right)^{H} \boldsymbol{P}^{H} \boldsymbol{H}^{*} \boldsymbol{x}_{m_{2}}^{\left(m_{1}\right)}\right\}-\left\|\boldsymbol{x}_{m_{2}}^{\left(m_{1}\right)}\right\|^{2}\right\} \\ \text { for all } m_{1} \neq i \text { and } m_{2} \neq j\end{array}\right.$

From (8), we are implied that the PSM shares the similarity of conventional multiuser transmitter preprocessing [12]. In terms of the transmitter preprocessing, the preprocessing matrix $\boldsymbol{P}$ should be designed in such ways that the desired receive antenna generates the maximal possible output, while minimizing the leak (interference) on the other receive antennas. Therefore, in this contribution, two typical transmitter preprocessing schemes, namely the transmitter zero-forcing (TZF) and transmitter minimum mean-square error (TMMSE) [12], are considered and the error performance of the PSM schemes is investigated, when these two transmitter preprocessing schemes are applied. 
Based on (5), an equivalent detection problem can be expressed as

$$
\boldsymbol{z}=\boldsymbol{W}^{H} \boldsymbol{y}=\boldsymbol{W}^{H}(\boldsymbol{H} \boldsymbol{x}+\boldsymbol{n})
$$

Then, with the aid of the equivalency existing between the transmitter preprocessing and multiuser detection, as detailed in $[12,14]$, we can readily show that, when the TZF is applied, we have $[12,14]$

$$
\boldsymbol{P}=\beta \boldsymbol{H}^{*}\left(\boldsymbol{H}^{T} \boldsymbol{H}^{*}\right)^{-1}
$$

where $\beta=\sqrt{M_{1} / \operatorname{Tr}\left(\left(\boldsymbol{H}^{T} \boldsymbol{H}^{*}\right)^{-1}\right)}$. By contrast, when the TMMSE is employed, we have $[12,14]$

$$
\begin{aligned}
\boldsymbol{P} & =\beta\left(\boldsymbol{H}^{*} \boldsymbol{H}^{T}+M_{1} \sigma^{2} \boldsymbol{I}_{N}\right)^{-1} \boldsymbol{H}^{*} \\
& =\beta \boldsymbol{H}^{*}\left(\boldsymbol{H}^{T} \boldsymbol{H}^{*}+M_{1} \sigma^{2} \boldsymbol{I}_{M_{1}}\right)^{-1}
\end{aligned}
$$

where, corresponding to (11) and (12), we have $\beta=\sqrt{M_{1} / \operatorname{Tr}\left(\left(\boldsymbol{H}^{*} \boldsymbol{H}^{T}+M_{1} \sigma^{2} \boldsymbol{I}_{N}\right)^{-2} \boldsymbol{H}^{*} \boldsymbol{H}^{T}\right)}$ and $\beta=\sqrt{M_{1} / \operatorname{Tr}\left(\left(\boldsymbol{H}^{T} \boldsymbol{H}^{*}+M_{1} \sigma^{2} \boldsymbol{I}_{M_{1}}\right)^{-2} \boldsymbol{H}^{T} \boldsymbol{H}^{*}\right)}$, respectively. Note that, in (11) and (12), the factor of $M_{1}$ is due to the on-off characteristic of the $M_{1}$ SSK invoked.

\section{Detection of PSM Signals}

Let us assume that $x_{j}^{(i)}$ was transmitted. Then, for the TZFassisted PSM, substituting $\boldsymbol{P}$ in the form of (10) into (5) yields the $M_{1}$-length decision variable vector

$$
\boldsymbol{y}=\beta \boldsymbol{x}_{j}^{(i)}+\boldsymbol{n}
$$

which can be expressed with respect to the $M_{1}$ receive antennas as

$$
\begin{aligned}
& y_{i}=\beta X_{j}+n_{i}, \\
& y_{m_{1}}=n_{m_{1}}, m_{1}=0,1, \ldots, M_{1}-1 ; m_{1} \neq i
\end{aligned}
$$

The decision variables in (14) show that only the one corresponding to the transmitted symbol contains both signal and noise, while all the others contain only noise.

For the TMMSE-based PSM, let in (11) $\boldsymbol{P}=\beta \tilde{\boldsymbol{P}}$, where $\tilde{\boldsymbol{P}}=\left[\tilde{\boldsymbol{p}}_{0}, \tilde{\boldsymbol{p}}_{1}, \cdots, \tilde{\boldsymbol{p}}_{M_{1}-1}\right]$. Then, when assuming that $\boldsymbol{x}_{j}^{(i)}$ was transmitted and submitting $\boldsymbol{P}=\beta \tilde{\boldsymbol{P}}$ into (5), we obtain the $M_{1}$-length decision variable vector

$$
\boldsymbol{y}=\beta \boldsymbol{H}^{T} \tilde{\boldsymbol{p}}_{i} \boldsymbol{x}_{j}^{(i)}+\boldsymbol{n}
$$

In more details, the $M_{1}$ decision variables generated by the $M_{1}$ receive antennas can be expressed as

$$
\begin{aligned}
& y_{i}=\beta \boldsymbol{h}_{i}^{T} \tilde{\boldsymbol{p}}_{i} X_{j}+n_{i}, \\
& y_{m_{1}}=\beta \boldsymbol{h}_{m_{1}}^{T} \tilde{\boldsymbol{p}}_{i} X_{j}+n_{m_{1}}, m_{1}=0, \ldots, M_{1}-1 ; m_{1} \neq i
\end{aligned}
$$

Furthermore, from (11), we can derive that, for $m_{1}=$ $0,1, \ldots, M_{1}-1$,

$$
\tilde{\boldsymbol{p}}_{m_{1}}=\left(\boldsymbol{H}^{*} \boldsymbol{H}^{T}+M_{1} \sigma^{2} \boldsymbol{I}_{N}\right)^{-1} \boldsymbol{h}_{m_{1}}^{*}
$$

Upon substituting them into (16), the $M_{1}$ decision variables can now be written as

$$
\begin{aligned}
y_{i}=\beta \boldsymbol{h}_{i}^{T}\left(\boldsymbol{H}^{*} \boldsymbol{H}^{T}+M_{1} \sigma^{2} \boldsymbol{I}_{N}\right)^{-1} \boldsymbol{h}_{i}^{*} X_{j}+n_{i}, \\
y_{m_{1}}=\beta \boldsymbol{h}_{m_{1}}^{T}\left(\boldsymbol{H}^{*} \boldsymbol{H}^{T}+M_{1} \sigma^{2} \boldsymbol{I}_{N}\right)^{-1} \boldsymbol{h}_{i}^{*} X_{j}+n_{m_{1}}, \\
m_{1}=0,1, \ldots, M_{1}-1 ; m_{1} \neq i
\end{aligned}
$$

which show that the decision variable matching to the transmitted symbol contains both the desired signal and Gaussian noise, while the other decision variables contain Gaussian noise and the interference resulted from the TMMSE preprocessing. According to [15], after the processing in MMSE principles, the resultant interference can be closely approximated as Gaussian noise.

Therefore, with the aid of the decision variables of (14) in the context of the TZF and that of (18) for the TMMSE, the PSM signals may be detected using the following approaches. First, when the detector is capable of tracking $\beta$ for the TZF and $\beta^{\prime}=\beta \boldsymbol{h}_{i}^{T}\left(\boldsymbol{H}^{*} \boldsymbol{H}^{T}+M_{1} \sigma^{2} \boldsymbol{I}_{N}\right)^{-1} \boldsymbol{h}_{i}^{*}$ for the TMMSE, a ML detector can be employed to detect the transmitted information according to the optimization problem

$$
\hat{m}, \hat{x}=\arg \min _{\boldsymbol{x}_{m_{2}}^{\left(m_{1}\right)} \in \mathcal{S}}\left\{\left\|\boldsymbol{y}-\alpha \boldsymbol{x}_{m_{2}}^{\left(m_{1}\right)}\right\|^{2}\right\}
$$

where $\alpha=\beta$ or $\beta^{\prime}$ for the TZF or TMMSE. Second, if the detector is unable to track the time-varying of $\beta$ or $\beta^{\prime}$, modified ML detector may be used. For example, the modified ML detector may have the same form as (19), but with $\alpha$ being replaced by the time-average of $\bar{\beta}$ or $\bar{\beta}^{\prime}$. Furthermore, the factor of $\beta$ invoked in transmitter preprocessing can be modified to a fix value. This can be achieved with the aid of the approximation $\boldsymbol{H}^{T} \boldsymbol{H}^{*} \approx N \boldsymbol{I}_{M_{1}}$ [13]. Using this approximation into $\beta=\sqrt{M_{1} / \operatorname{Tr}\left(\left(\boldsymbol{H}^{T} \boldsymbol{H}^{*}\right)^{-1}\right)}$ yields $\beta=\sqrt{N}$ for the TZF. Similarly, with the aid of the above approximation, we can obtain from $\beta=\sqrt{M_{1} / \operatorname{Tr}\left(\left(\boldsymbol{H}^{T} \boldsymbol{H}^{*}+M_{1} \sigma^{2} \boldsymbol{I}_{M_{1}}\right)^{-2} \boldsymbol{H}^{T} \boldsymbol{H}^{*}\right)}$ that $\beta=\left(N+M_{1} \sigma^{2}\right) / \sqrt{N}$ for the TMMSE. Note that, even using the fixed value of $\beta=\sqrt{N}$ or $\beta=\left(N+M_{1} \sigma^{2}\right) / \sqrt{N}$, the transmitter still satisfies the power constraint on transmission, if the transmission duration is long enough.

Furthermore, when the detector is unable to track the timevarying behavior of $\beta$ or $\beta^{\prime}$, the sub-optimal detector as following may also be employed for detection. The detection process can be divided into two steps. First, the pre- $M_{1}$ SSK signal is non-coherently detected based on the decision variables $\left\{\left|y_{0}\right|^{2},\left|y_{1}\right|^{2}, \cdots,\left|y_{M_{1}-1}\right|^{2}\right\}$ formed from (14) or (18). The maximum of $\left\{\left|y_{0}\right|^{2},\left|y_{1}\right|^{2}, \cdots,\left|y_{M_{1}-1}\right|^{2}\right\}$ is selected and its index is mapped to an integer value in $\left[0, M_{1}-1\right]$, which represents the symbol conveyed by the pre- $M_{1}$ SSK modulation. After the pre- $M_{1}$ SSK demodulation, the APM signal (i.e., $X_{j}$ in (14) or (18)) can then be detected using the conventional demodulation approaches based on the output of the receive antenna identified by the pre- $M_{1}$ SSK demodulation.

Let us below provide some figures for illustrating the achievable BER performance of the PSM schemes. 


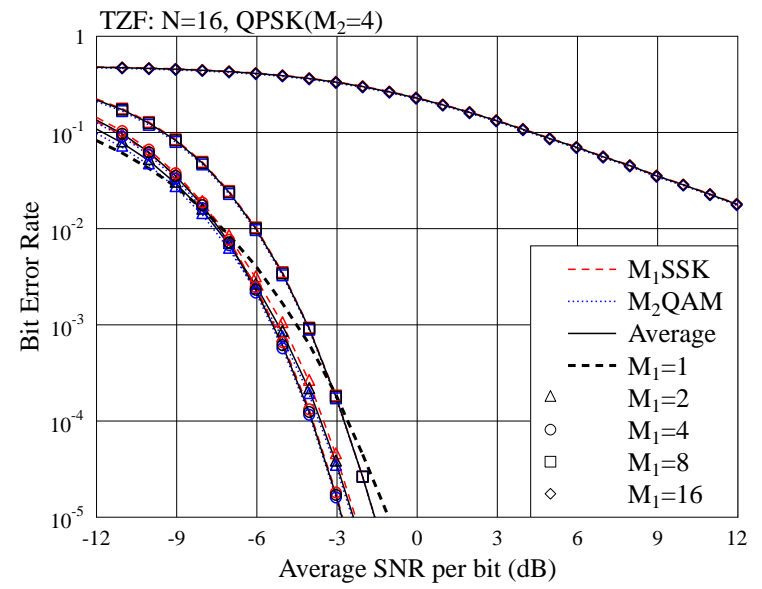

Fig. 1. BER versus average SNR per bit performance of the PSM system with TZF-assisted preprocessing.

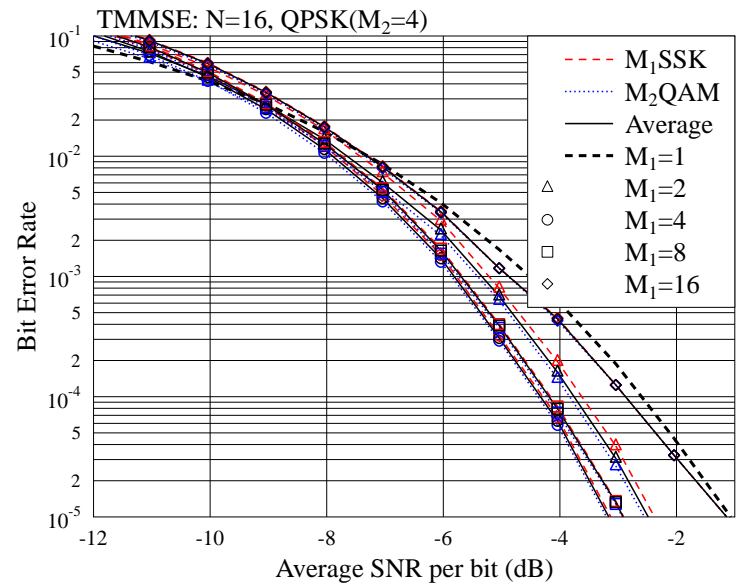

Fig. 2. BER versus average SNR per bit performance of the PSM system with TMMSE-assisted preprocessing.

\section{Performance Results}

In this section, we provide a range of BER performance results for the PSM with various combinations of $M_{1}$ SSK and $M_{2}$ QAM. Due to limited space, here we focus only on the first type detector as stated in Section IV, which is assumed can track the time-varying factor $\beta$ or $\beta^{\prime}$. BER performance of PSM using the other two types of detectors will be provided in our related publications elsewhere. In this section, we concern the individual BER of both the $M_{1}$ SSK and $M_{2}$ QAM involved, as well as the overall average BER of the PSM. Note that, in the following figures, $M_{1}=1$ or $M_{2}=1$ indicates that no $M_{1} \mathrm{SSK}$ or $M_{2} \mathrm{QAM}$ is involved. In other words, $M_{1}=1$ corresponds to the pure MQAM, while $M_{2}=1$ to the pure MSSK modulation.

Figs. 1 and 2 illustrate the BER performance of the PSM with respect to the $M_{1}$ SSK modulation with various values for $M_{1}$, when the system employs $N=16$ transmit antennas to transmit QPSK constellation. From the results of Figs. 1 and 2, we can have the following observations. First, given the APM of QPSK

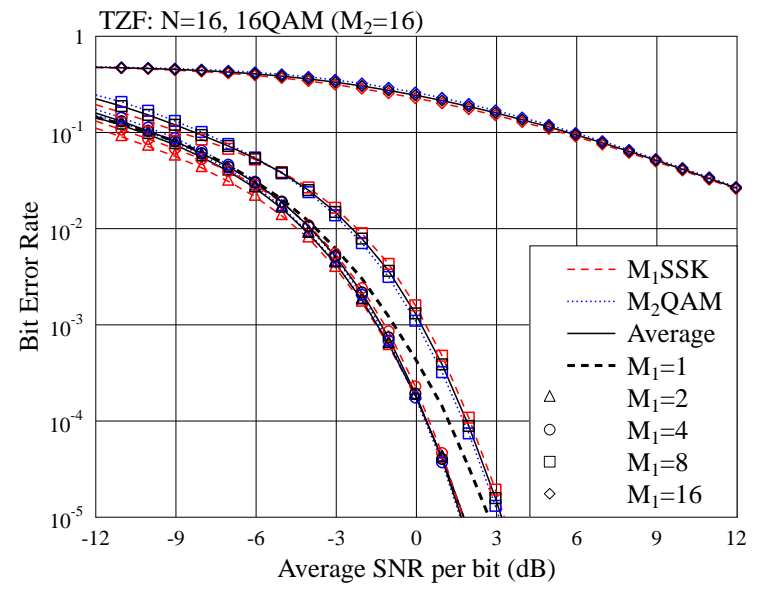

Fig. 3. BER versus average SNR per bit performance of the PSM system with $\mathrm{TZF}$-assisted preprocessing.

and at a given SNR per bit, there exists an optimal value of $M_{1}$, which generates the best BER performance. For example, at the SNR per bit of $-4 \mathrm{~dB}$, the system using both the TZF and TMMSE is capable of attaining the best BER performance, when $M_{1}=4$, i.e., when using 4 SSK modulation. Second, for some $M_{1}$ values, such as $M_{1}=2$, 4, employing $M_{1}$ SSK modulation is capable of enhancing the QPSK's BER performance, in addition to the increased throughput provided by the $M_{1}$ SSK modulation. For example, as shown in Fig. 2, without using $M_{1}$ SSK corresponding to $M_{1}=1$, the system achieves the throughput of 2 bits/symbol from the QPSK with the BER of $10^{-5}$ at a SNR per bit of about $0 \mathrm{~dB}$. When using both 4 SSK and QPSK, promisingly, the system achieves a throughput of 4 bits/symbol with the same BER of $10^{-5}$ but at a SNR per bit of about $-3 \mathrm{~dB}$. Hence, due to the employment of $4 \mathrm{SSK}$, the system is capable of obtaining about $3 \mathrm{~dB}$ of SNR gain, in addition to doubling the throughput. From Fig. 1, we can also draw similar observations, although the SNR gain is lower than $3 \mathrm{~dB}$. Third, when the value of $M_{1}$ is relatively low, such as $M_{1} \leq 4$, the average BER performance of the PSM is seems dominated by the $M_{1}$ SSK. In contrast, when the value of $M_{1}$ is sufficiently high, the average BER performance of the PSM is then dominated by the QPSK modulation. Finally, when comparing Fig. 1 for TZF and Fig. 2, we can see that the TMMSE scheme outperforms the TZF scheme, especially, when $M_{1}$ is high, such as $M_{1}=N=16$. This observation implies that the TZF scheme also suffers from the problem of noise amplification due to the ZF operation, which significantly degrades the BER performance as it does in ZF-based multiuser detection and transmitter preprocessing [12].

Figs. 3 and 4 depict the BER performance of the PSM with respect to the $M_{1}$ SSK modulation having various values for $M_{1}$, when the system employs $N=16$ transmit antennas and 16QAM in addition to the $M_{1}$ SSK. The parameters and assumptions used for Figs. 3 and 4 were the same as that for Figs. 1 and 2, except that the QPSK modulation considered in Figs. 1 and 2 was replaced by 16QAM. Explicitly, from Figs. 3 and 4 , we can draw similar conclusions as those drawn from 


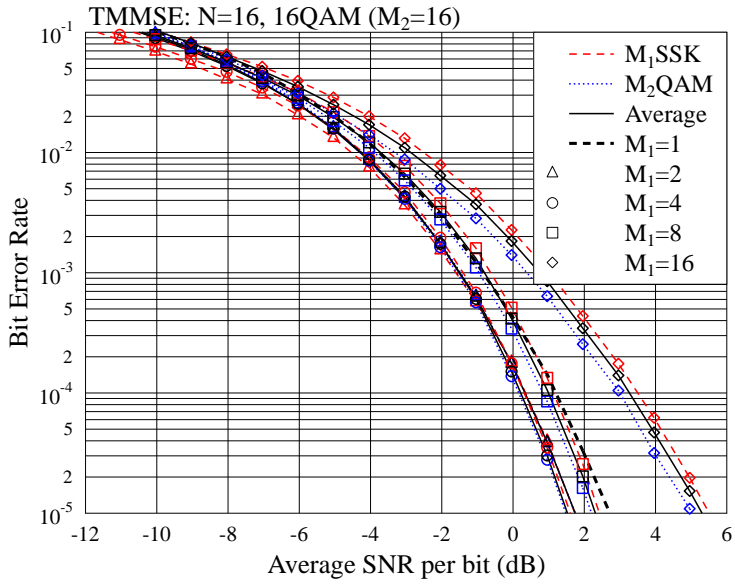

Fig. 4. BER versus average SNR per bit performance of the PSM system with TMMSE-assisted preprocessing.

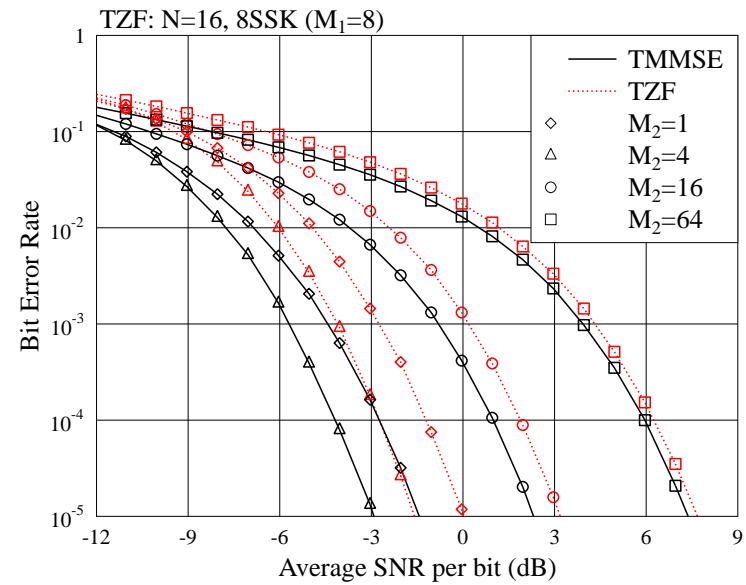

Fig. 5. Comparison of BER versus average SNR per bit performance of the PSM system with TMMSE- and TZF-assisted preprocessing.

Figs. 1 and 2. However, given a preprocessing scheme, when all the other parameters except the APM (QPSK v.s 16QAM) are the same, the BER performance in Fig. 1 or Fig. 2 is better than that in Fig. 3 or Fig. 4, due to the well-known fact that the QPSK outperforms the 16QAM in terms of BER performance.

In Fig. 5, we illustrate the BER performance of the PSM system using 8SSK and the APM schemes of QPSK, 16QAM and 64QAM, when the PSM system employs $N=16$ transmit antennas. Furthermore, as mentioned previously, $M_{2}=1$ corresponds to the PSM system without using APM. From the results of Fig. 5, we can have some typical observations, such as that TMMSE outperforms TZF, that BER performance degrades as the value of $M_{2}$ increases, etc. An important observation is that, as seen in Fig. 5, employing the APM may aid the $M_{1}$ SSK to enhance its BER performance, in addition to the added throughput by the APM. Taking the TMMSE as an example, as seen in Fig. 5, without employing the APM, the 8SSK achieves the BER of $10^{-5}$ at about $-1.5 \mathrm{~dB}$. How- ever, when the PSM system uses both the 8SSK and QPSK $\left(M_{2}=4\right)$, it can achieve the BER of $10^{-5}$ at about $-3 \mathrm{~dB}$. Hence, in addition to the extra throughput of 2 bits/symbol, the PSM system is also capable of obtain about $1.5 \mathrm{~dB}$ of SNR gain.

\section{CONCLUSIONS}

In this paper, we have proposed a transmitter preprocessing aided SM scheme, namely the PSM scheme, for the MIMO systems operated under the CSIT mode. The preprocessing optimization and signal detection have been analyzed. Both the TZF and TMMSE preprocessing schemes have been investigated. Three types of strategies have been proposed for detection of the PSM signals. Finally, the achievable BER performance of the PSM under different system settings has been demonstrated. Our analysis and performance results show that the PSM can provide an alternative way for signal transmission in MIMO systems. In PSM, when appropriately designed, the embedded $M_{1}$ SSK modulation and APM can help each other to achieve the overall BER performance, which is better than the BER performance of the pure APM or that of the pure $M_{1}$ SSK modulation invoked in the PSM.

\section{REFERENCES}

[1] Y. Chau and S.-H. Yu, "Space modulation on wireless fading channels," in Proc. of IEEE 54th VTC'01 (Fall). IEEE, September 2001, pp. 1668 $-1671$.

[2] S. Ganesan, R. Mesleh, H. Haas, C. Ahn, and S. Yun, "On the performance of spatial modulation of OFDM," in Proc. 40th Asilomar Conf. Signals, Syst. Comput., Oct. 29 - Nov. 1 2006, pp. 1825 - 1829.

[3] R. Mesleh, H. Haas, C. Ahn, and S. Yun, "Spatial modulation - a new low-complexity spectral efficiency enhancing technique," in Proc. Chinacom'2006, October 2006, pp. 1-5.

[4] R. Mesleh, H. Haas, S. Sinanovic, C. Ahn, and S. Yun, "Spatial modulation," IEEE Transactions on Vehicular Technology, vol. 57, no. 4, pp. 2228 - 2241, July 2008.

[5] J. Jeganathan, A. Ghrayeb, L. Szczecinski, and A. Ceron, "Space shift keying modulation for MIMO channels," IEEE Transactions on Wireless Communications, vol. 8, no. 7, pp. 3692 - 3703, July 2009.

[6] M. Renzo and H. Haas, "Improving the performance of space shift keying (SSK) modulation via opportunitic power allocation," IEEE Communications Letters, vol. 14, no. 6, pp. 500 - 502, June 2010

[7] — "A general framework for performance analysis of space shift keying (SSK) modulation for MISO correlated Nakagami- $m$ fading channels," IEEE Transactions on Communications, vol. 58, no. 9, pp. September 2010.

[8] J. Jeganathan, A. Ghrayeb, and L. Szczecinski, "Spatial modulation: Optimum detection and performance analysis," IEEE Communications Letters, vol. 12, no. 8, pp. 545 - 547, August 2008.

[9] G. J. Foschini, "Layered space-time architecture for wireless communication in a fading environment when using multiple antennas," Bell Labs Technical Journal, pp. 41-59, Autumn 1996.

[10] V. Tarokh, H. Jafarkhani, and A. R. Calderbank, "Space-time block coding for wireless communications: performance results," IEEE Journal on Selected Areas in Communications, vol. 17, no. 3, pp. 451-460, March 1999.

[11] V. Tarokh, A. Naguib, N. Seshadri, and et al., "Space-time codes for high data rate wireless communication: Performance criteria in the presence of channel estimation errors, mobility, and multiple paths," IEEE Transactions on Communications, vol. 47, no. 2, pp. 199-207, Febuary 1999.

[12] L.-L. Yang, Multicarrier Communications. Chichester, United Kingdom: John Wiley, 2009.

[13] I. E. Telatar, "Capacity of multiantenna Gaussian channels," European Transactions on Telecommunications, vol. 10, no. 6, pp. 585-595, Nov./Dec. 1999.

[14] L.-L. Yang, "Design linear multiuser transmitters from linear multiuser receivers," in IEEE ICC'2007. IEEE, June 2007.

[15] H. V. Poor and S. Verdu, "Probability of error in MMSE multiuser detection," IEEE Transactions on Information Theory, vol. 43, no. 3, pp. 858-871, May 1997. 